\title{
Bench measurements of Coupling Impedance of AGS Booster Components*
}

\author{
A. Ratti and T. J. Shea \\ Brookhaven National Laboratory \\ Upton NY, 11973
}

BNL- -45476

DE91 014805

\section{Abstract}

Quantifying instability thresholds for modem synchrotrons and storage rings requires some knowledge of the accelerator's coupling impedance. To this end, the wire technique has been implemented to measure the longitudinal coupling impedance of AGS Booster devices. The techniques are being refined to allow measurement of RHC devices at higher frequencies. All the measurements are performed using an HP8753 Network Analyzer controlled via GPIB by a Macintosh computer. The computer provides an environment for automated data acquisition. data analysis, and report generation. Resistive matches between the $50 \Omega$ analyzer cables and the $300 \Omega$ pipe-and-wire structure allow the use of a simple response calibration in the measurement of S21 to $400 \mathrm{MHz}$. Results from ferrite loaded if cavities, position monitors and kickers are presented.

\section{INTRODUCTION}

The AGS Booster is an injector for the AGS and RHIC complex. It will run high intersity protons in order to increase the beam current available to AGS experimenters, as well as heavy ions both for fixed target experiments and eventually for injection in the RHIC. Since over $10^{\wedge} 13$ protons will be accelerated in each Booster pulse, resulting in high currents on the walls, some consideration has been given to the problem of coupling impedances and the related collective instabilities. This lead to the development of a lab setup capable of measuring the coupling impedance of various devices before installation in the Booster ring. Due to the time constraints of this effort, only the longitudinal effects have been measured; efforts for acquiring information on transverse impedances are planned for the future, both for spare Booster devices and RHIC prototypes.

\section{MEASUREMENT TECHNIQUE}

The wire method was adopted with resistive matches used to adapt the typical $50 \Omega$ measurement equipment to the $300 \Omega$ pipe-and-wire geometry. This simple geometry is detailed in Fig. 1. With non-reflective resistive matches, a full two port calibration of the network analyzer is not necessary and the simple response correction suffices. This type of resistive match shows acceptable performance for frequencies up to about $400 \mathrm{MHz}$.

All measurements were done in a frequency domain in order to take advantage of the large dynamic range available with modem network analyzers. The technique is based on measurements of the transmission parameter S21 of the

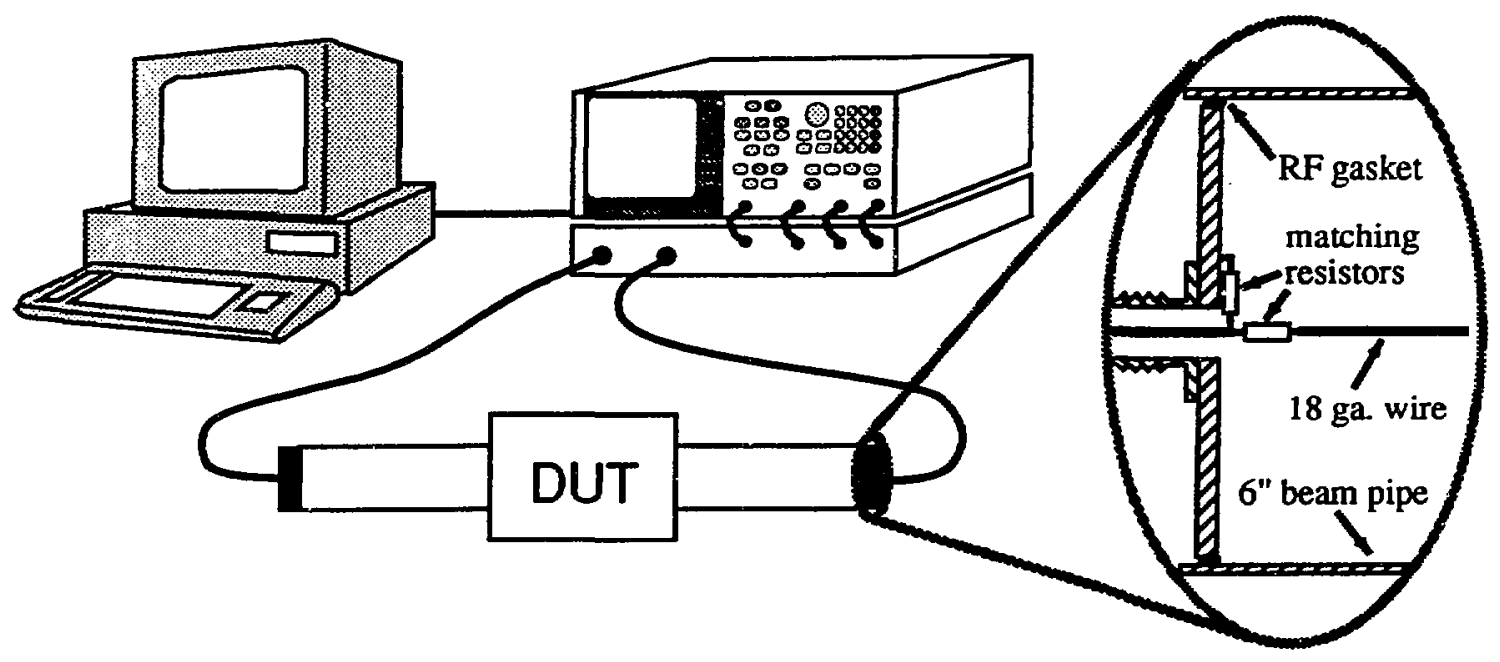

Figure 1. Setup for measuring longitudinal coupling impedance

-Work supported by the US Department of Energy MASTER 
scattering matrix, from which the corresponding coupling impedance can be calculated. The characteristic imnedance of the measuring apparatus was chosen to be 3002 as set by practical limitations in the thickness of the inner conductor (wire) of the coaxial structure and by the 6 inch beam pipe diameter chosen for the Booster. From the measured scattering

$$
Z=2 Z_{0} \frac{1-S_{21}}{S_{21}}
$$

parameter, the longitudinal coupling impedance $\mathrm{Z}$ can be calculated using [1] :

Particular attention has been paid to keeping the measurement conditions as close as possible to those existing during the device's operation in the ring. To demonstrate the importance of this, the pick up electrode has been measured both with the front end electronics connected and with the two ports open, showing a dramatic change in the behavior of the impedance.

\section{DESCRIPTION OF THE APPARATUS}

The setup is shown in Fig. 1. A Macintosh computer, running LabVIEW routines interfaces via GPIB-to an HP 8753 network analyzer. The calibration of this instrument is one of the most important tasks, and there are two possibilities available in this setup. The instrument can be calibrated on a straight pipe, assumed as a reference "thro'ugh" to record the power dissipated on the matching resistors and the effect of the setup's electrical length. As an altemative, the analyzer may be calibrated on a standard $50 \mathrm{CHM}$ "through", deferring the subtraction of the reference pipe to a later moment. In this case, the calibration is completed during the processing of the data in the computer. These two techniques are intrinsically identical and only practical considerations lead to the most convenient choice.

The Macintosh is also used to perform the calculation of the coupling impedance, which is done in a math package with complex algebra capabilities. Impedance is then available in real and imaginary form, or in terms of amplitude and phase.

\section{MEASUREMENTS RESULTS}

\section{Cavity}

The cavity was a very important reference for testing the setup, since it was possible to check the measurement results with other system tests. These system tests, however, were restricted to the operating frequency band. The device measured is the Band III If cavity, which is a double gap ferrite loaded coaxial resonator [2]. It is tunable over a frequency range from 2.5 to $4.2 \mathrm{MHz}$ by changing the permeability of the ferrite with a biasing field. It is driven with a single ended power amplifier, and internally cross coupled with a "figure of eight" winding in order to guarantee its push

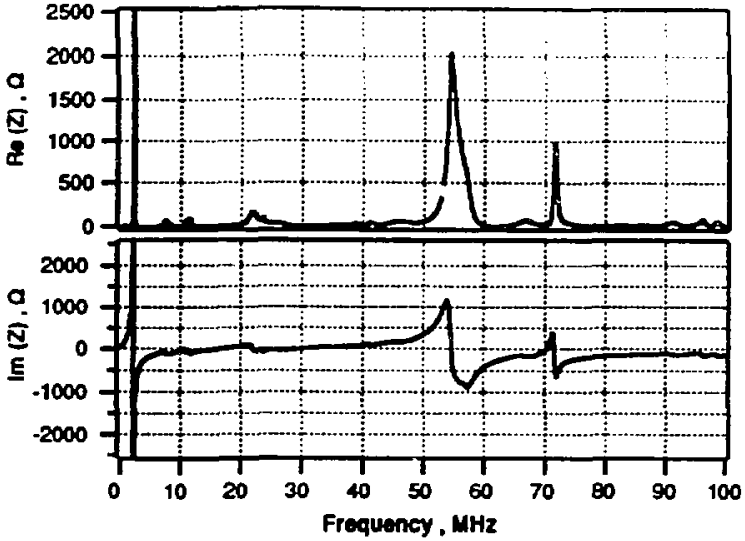

Figure 2. Rf cavity measurement

pull operation. Even though the power amplifier plays an important role in the loaded cavity, it was not practical to measure the system at full power. Nevertheless the results have been successfully compared to values obtained by extrapolation from the system calorimetry test data. The effects of the tuning currents have also been investigated and showed very little or no effect on the resonances found above the fundamental. The results of the measurements of one of the two Band III cavities used in the Booster are shown in Fig. 2.

\section{PUE}

The beam pick up electrode (PUE) is a cylinder split at a $45 \mathrm{deg}$ angle. A total of 48 of these position monitors are used in the Booster ring [3]. The measurement setup in this case is particularly critical, since the behavior of the impedance is severely affected by the front end electronics. Figure 3 shows

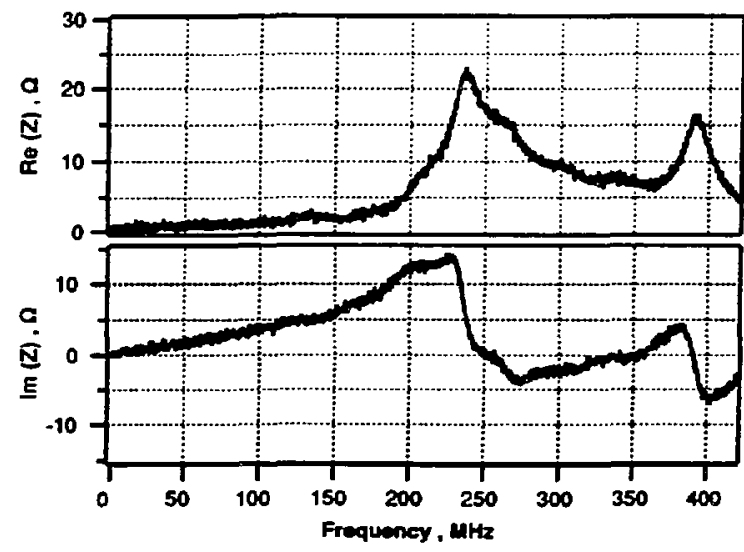

Figure 3. PUE with actual front end connected 


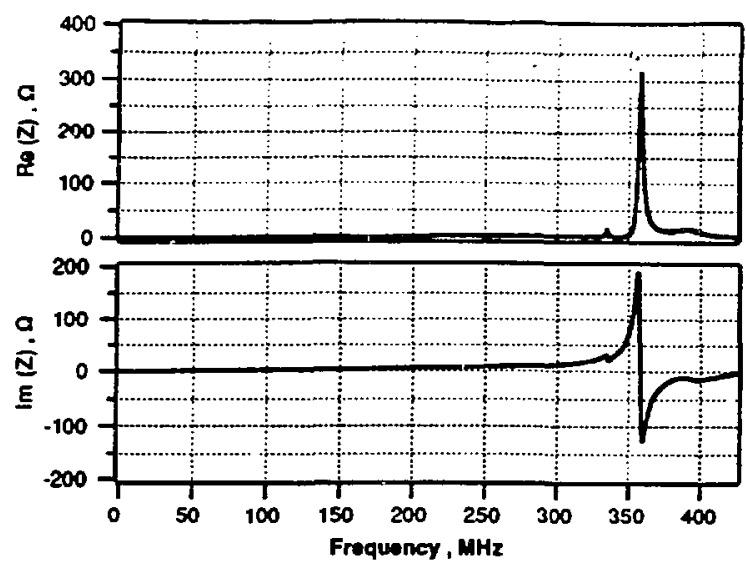

Figure 4. PUE with ports open

the result of a coupling impedance measurement with the position monitor setup that is being used in the Booster ring. In this case, the PUE is connected to a series resistor followed by $10 \mathrm{ft}$ of matched cables leading to the front end electronics. However, if the PUE is left unterminated, the impedance behaves in a totally different manner, as shown in Figure 4.

\section{Kicker}

The Booster injection kicker measured here is a ferrite device with the azimuthal flux path split by two copper plates in order to reduce the beam induced effects [4]. The effectiveness of this design has been confirmed by the results of high power tests with and without the copper split. Figure 5 shows the meastired longitudinal impedance of this device.

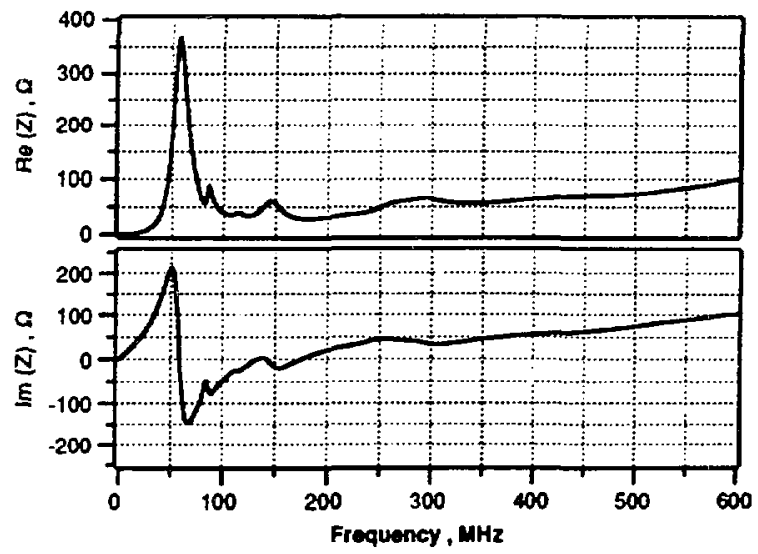

Figure 5. Kicker measurement

\section{STATUS}

In the future, most of the vacuum components for RHIC will be measured. For these devices, it will be important to measure at higher frequencies. An improved version of the resistive matches is under development and the time domain gating technique will also be implemented. In parallel with these experiments, some studies are underway with the available computer codes.

\section{ACKNOWLEDGMENTS}

The authors would like to thank some of people who shared support and enlightenment: we are grateful to Peter Cameron and Ken Rogers for providing devices for each measurement, to Chris Degen for writing the data acquisition software, to F. Caspars, G. Lambertson and E. Raka for very useful discussions.

\section{REFERENCES}

[1] H. Hahn and F. Pedersen, "On coaxial wire measurements of the longitudinal coupling impedance", BNL report BNL-50870, April 1978

[2] R. T. Sanders, et al.. The AGS Booster high frequency rf system", these proceedings

[3] D. J. Ciardullo, et al., "The AGS Booster beam position monitor system", these proceedings

[4] W. K. Van Asselt and Y. Y. Lee, "Beam induced heating of ferrite magnets", these proceodings

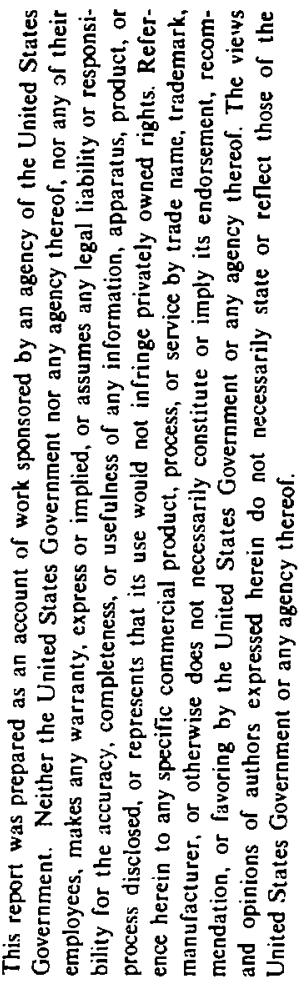

